\title{
Mitochondrial DNA in Fresh versus Frozen Embryo Culture Media of Polycystic Ovarian Syndrome Patients Undergoing Invitro Fertilization: A Possible Predictive Marker of a Successful Pregnancy
}

This article was published in the following Dove Press journal:

Pharmacogenomics and Personalized Medicine

\begin{abstract}
Ghadir A Sayed (ID'
Hussein A Al-Sawaf'

Ahmed H Al-Sawaf ${ }^{2}$

Mohamed Saeid ${ }^{3}$

Ahmed Maged (D) ${ }^{2}$

Iman Hassan Ibrahim (iD) 4

'Department of Biochemistry, Faculty of Pharmacy, Egyptian Russian University, Cairo, Egypt; ${ }^{2}$ Department of Obstetrics and Gynecology, Faculty of Medicine, Cairo University, Cairo, Egypt; ${ }^{3}$ Ganna Center for IVF, Cairo, Egypt; ${ }^{4}$ Department of Biochemistry, Faculty of Pharmacy (Girls), Al-Azhar University, Cairo, Egypt
\end{abstract}

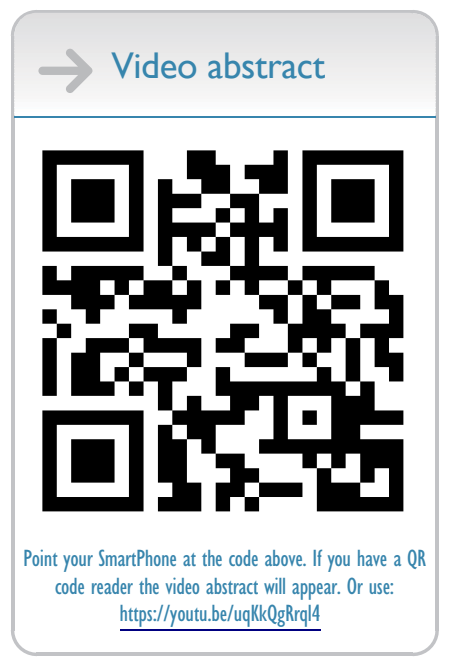

Correspondence:Ghadir A Sayed Department of Biochemistry, Faculty of Pharmacy, Egyptian Russian University, Cairo I2I II, Egypt

Tel +20IIII 826634

Fax +2028609II7

Email ghadir-ali@eru.edu.eg
Purpose: Frozen embryos transfer (ET) may improve the live-birth and reduce rates of ovarian hyperstimulation in polycystic ovary syndrome (PCOS) patients. Morphological criteria are the classical way for embryo selection, yet recently, many biochemical and genetic markers have been developed. This study aimed to compare fresh and frozen ET using the mtDNA/gDNA ratio of embryo secretome and the possibility of using this ratio as a predictive marker of PCOS pregnancy rate.

Subjects and Methods: One hundred PCOS patients undergoing IVF were chosen according to Rotterdam criteria and divided into two groups. Group I (50 with fresh ET), group II (50 with frozen ET), and otherwise 33 apparently healthy women as a control group with fresh ET. We then carried out absolute quantification of embryo culture media mtDNA and gDNA by real-time PCR.

Results: mtDNA/gDNA ratio was significantly low in PCOS embryo culture media in comparison with control. Additionally, while the mtDNA/gDNA ratio was significantly high in pregnant PCOS embryo culture media, it was high, though not statistically significant, in the fresh ET than frozen ET group. mtDNA/gDNA ratio sensitivity and specificity in PCOS embryo culture media as a predictive value of pregnancy rate were $(86 \%$ and $96 \%$, respectively).

Conclusion: mtDNA/gDNA ratio measurement in PCOS embryo culture media is a novel marker that can be clinically applied as a predictive value of the quality of the morphologically good embryo.

Keywords: frozen embryos transfer, fresh embryos transfer, mtDNA, polycystic ovary syndrome

\section{Introduction}

PCOS is the most common endocrinopathy disorder of women of reproductive age. It is characterized by hyperandrogenism, ovulatory dysfunction, and polycystic ovaries. ${ }^{1}$ Anovulation is one of the major causes of infertility as only $7-18 \%$ of PCOS patients are ovulatory. ${ }^{2}$ Assisted reproductive technology is used to improve pregnancy rates. It involves monitoring and stimulating the patient's ovulatory process to obtain the gametes which can be fertilized invitro. After fertilization, morphologically sound good embryos are transferred into the uterine cavity. ${ }^{3}$ 
Infertile women with PCOS may have the needed number of retrieved oocytes in IVF cycles; however, most of these oocytes have poor quality. ${ }^{4}$ Additionally, Ovarian Hyperstimulation Syndrome (OHSS) is considered one of the most serious complications of IVF. ${ }^{5}$ It was reported that controlled ovarian stimulation is associated with deleterious effects on endometrial receptivity due to high levels of estrogen and progesterone during the follicular phase compared to natural cycles. ${ }^{6}$ The risk of OHSS is, reportedly, increased after the transfer of fresh embryo and pregnancy occurrence in cycles with ovarian stimulation. $^{7}$ However, transferring frozen-thawed embryos in unstimulated cycles eliminates the risks of OHSS and, subsequently, increases pregnancy rates ${ }^{8}$ Many studies have compared clinical outcomes between fresh and frozen ET strategies. Nonetheless, the efficiency and safety of these two methods remain unclear. While Chen et $\mathrm{al}^{9}$ and Coates et $\mathrm{al}^{10}$ studies reported a significant increase in the live birth rate in the frozen ET group. Also, some studies showed that the freeze-all strategy increases pregnancy rates and minimizes the risk of OHSS. ${ }^{11,12}$ While, another study showed a lower live birth rate in frozen ET cycles when compared to fresh cycles due to freeze-thaw process-related embryo damage. ${ }^{13}$

Although non-invasive evaluation of embryo viability is, ideally, based on morphologic and morphometric grading systems, they are affected by the variability of individual judgment. ${ }^{14}$ Embryo morphology does not reflect functional status, as $30-50 \%$ of morphologically good embryos might be chromosomally abnormal. ${ }^{15}$ Analysis of metabolic parameters in embryo culture media is used as non-invasive embryo selection methods, such as glucose, lactate, pyruvate, amino acids, and oxygen consumption. ${ }^{16}$

mtDNA is a double-stranded circular DNA molecule of approximately $16.5 \mathrm{~kb}$ that encodes 13 subunits of the respiratory chain complexes, which use oxidative phosphorylation for the production of ATP in the cell. ${ }^{17}$ This genome also includes 2 rRNAs, 22 tRNAs that are needed for mitochondrial protein synthesis. This in addition to a non-coding region, D-loop, which consists of 1122 base pairs acting as a promoter of mtDNA, and contains essential transcription and replication elements. ${ }^{18}$ mtDNA is not transmitted in a pattern of Mendelian inheritance, an oocyte cytoplasm passes it from one generation to the next. ${ }^{19}$ Mutations in the non-coding region, D-loop, may have a direct, or indirect role in inducing repeated pregnancy loss. ${ }^{20}$ Thus, mtDNA/gDNA ratio in the embryo secretome, with morphological grading, can be used as a non-invasive, early biomarker for the identification of viable embryos. $^{21}$

The purpose of this study is to Quantify mtDNA/ gDNA ratio at embryo culture media as non-invasive markers of pregnancy rate in PCOS patients and compare this ratio with the control group. Furthermore, this study aims to compare the mtDNA/gDNA ratio in fresh and frozen samples of embryo culture media of PCOS patients' undergoing IVF and to calculate the specificity and sensitivity of this ratio as a biochemical marker of good embryo quality.

\section{Subjects and Methods Ethical Approval}

This study was carried out in accordance with the Declaration of Helsinki for experiments involving humans, and its protocol was reviewed and approved by Al-Azhar University Faculty of Pharmacy (Girls) Institutional Review Board (approval no. 134). Written informed consent was submitted by all subjects when they were enrolled. The clinical trial registration NCT03820167.

\section{Subjects}

This study included 100 PCOS women who were diagnosed according to Rotterdam criteria and had two of the following: clinical and/or biochemical hyperandrogenism, oligo-ovulation or anovulation and polycystic ovaries (the presence of 12 or more follicles measuring between 2 and $9 \mathrm{~mm}$ in diameter and/or an increased ovarian volume of greater than $10 \mathrm{~cm}^{3}$ at least in one ovary). ${ }^{22}$ They were scheduled for ICSI (Intracytoplasmic Sperm Injection) and were chosen according to a strict selection criterion: Inclusion criteria included age (20-35 yrs.), BMI $\leq 25$ to eliminate hormonal dysregulation of obesity, Nulligravida, and primary infertility. Exclusion criteria: known medical problems like diabetes mellitus, hypertension, cardiac, hepatic, or renal problems, special habits of medical importance as smoking and other endocrinopathies.

Patients were classified into two groups: group I (50 PCOS women with fresh ET strategy), and group II (50 PCOS women with frozen ET strategy), and otherwise 33 healthy women undergoing IVF due to unexplained infertility. All samples were collected in 2018-2019 from Ganna Center for Invitro Fertilization, Giza, Cairo. PCOS patients with high risk for OHSS (estradiol $>4000$ $\mathrm{pg} / \mathrm{mL}$ ) were scheduled for frozen ET whereas patients 
with lower risk (estradiol $<4000 \mathrm{pg} / \mathrm{mL}$ ) were scheduled for fresh ET and a group of two morphologically good embryos are chosen for fresh ET and the remaining morphologically good embryos were frozen in day 3 .

\section{In vitro Fertilization Protocol, Embryo Culture, and Medium Collection}

All included subjects received an antagonist protocol. This protocol included ovarian stimulation by recombinant FSH (r-FSH) (Gonal f, Merk, UK) and HMG (Merional, IBSA, Switzerland). The ovarian response to stimulation was monitored by quantifying serum estradiol levels. The ovarian stimulation length was $13.45 \pm 0.12$ days and the total gonadotropin dose was 1930.7 $\pm 31.08 \mathrm{IU} / 1($ mean \pm SEM). When at least three Graffian follicles reached $18 \mathrm{~mm}$ in mean diameter, ovulation dual trigger therapy was used in fresh embryo transferred group which included administration of gonadotropin agonist trigger $(0.1 \mathrm{mg} \mathrm{sc}$ (Decapeptyl; Ipsen S.P.A., Milan, Italy)) and injection of hCG (Choriomon, IBSA, Switzerland) (5000 IU/1). While in the frozen embryo transferred group, the ovulation was triggered by the administration of decapeptyl only. The estradiol level at ovulation triggering was measured. Transvaginal oocyte retrieval (oocyte pick-up) was initiated 34-36 hours following ovulation triggering. Retrieved oocytes were rinsed, graded, and placed in the clinic's routine retrieval media for incubation and fertilization using the ICSI method. Day 3 ET was chosen in fresh ET as transferring embryo at day 5 may not provide any additional benefit as regards to clinical pregnancy rate. ${ }^{23}$ Embryos scheduled for freezing were cryopreserved by the verification method using liquid nitrogen (open system) for less than 1 year. Day 4 ET was chosen in frozen ET: frozen day 3 embryos were thawed 1 day before transfer and left to cleave in the incubator then only two morphologically good embryos were chosen for ET on day 4 . The embryo scoring system was used to choose the morphologically good embryos as following: (a) embryos of grade two ( $\geq 5$ cells, blastomeres of equal size, and less than $30 \%$ cytoplasmic fragmentation) according to cleavage stage scoring system were chosen for day 3 ET in fresh ET group and (b) embryos of incomplete compaction (some of the blastomeres are distinct as individual cells while other areas as amorphous mass) were chosen for day 4 ET in frozen ET group. ${ }^{24}$

The media of fresh and frozen embryos $(20 \mu \mathrm{L})$ were immediately collected into sterile, DNA-, DNase-, RNase-,
Polymerase Chain Reaction (PCR) inhibitors-free tubes (Eppendorf, Hamburg, Germany) and stored at $-20^{\circ} \mathrm{C}$ until nucleic acid purification. Appropriate precautions were taken to prevent contamination of samples by extraneous cells or DNA. During each experimental step, laboratory personnel wore gloves and coat, and physical isolation was guaranteed by working in clean air hoods.

\section{DNA Extraction and Purification}

DNA was concentrated and purified from embryo culture media using a spin column-based method (GeneJETTM Genomic DNA Purification Kitcat\#K0722) (ThermoScientific, California) according to the manufacturer's instructions. The reagent was adjusted to compensate for the reduced volume of the culture medium sample.

\section{Preparation of Standard for Absolute Quantification}

DNA isolated from control samples was used for the amplification of mitochondrial and nuclear genes with appropriate primers hMito for mtDNA and $\mathrm{hB} 2 \mathrm{M}$ for gDNA. ${ }^{25}$

The reaction mixture was prepared by adding $10 \mu \mathrm{L}$ of QuantiTect SYBR Green PCR Master Mix, $0.5 \mu \mathrm{L}$ of forward, $0.5 \mu \mathrm{L}$ reverse primer $(0.5 \mu \mathrm{M}$ of each), and $4 \mu \mathrm{L}$ RNase-free $\mathrm{H}_{2} \mathrm{O}$. Then, we added 1-2 $\mu \mathrm{L}$ of DNA template (1 $\mu \mathrm{g}$ of DNA). We performed PCR, using Thermal cycler (Applied Biosystem Step One PlusTM), with an initial denaturation at $95^{\circ} \mathrm{C}$ for 5 min $(1$ cycle), followed by denaturation at $95^{\circ} \mathrm{C}$ for $10 \mathrm{~s}$, annealing at $60^{\circ}$ $\mathrm{C}$ for $30 \mathrm{~s}$, and extension $40^{\circ} \mathrm{C}$ for $30 \mathrm{~s}$. The PCR products were visualized by electrophoresing on a $2 \%$ agarose gel. The bands were excised and DNA was purified and eluted in 20-30 $\mu \mathrm{L}$ of elution buffer. The concentration of each PCR product was determined by using the Nano-Drop and copy number per $1 \mu \mathrm{L}$ calculated as follows:

For hMito: Copy number $=\mathrm{C} \times 7.18 \times 10^{9} / \mu \mathrm{L},(1)$

For hB2M: Copy number $=\mathrm{C} \times 4.87 \times 10^{9} / \mu \mathrm{L},(2)$

where $\mathrm{C}=$ concentration of DNA in $n g / \mu \mathrm{L}$.

The value of $7.18 \times 10^{9}$ is derived using the following formula: Avogadro's constant $\left(6.02 \times 10^{23}\right) / \mathrm{PCR}$ product size $(127 \mathrm{bp}$ for hMito $) \times$ molecular weight of ds DNA (660 Da/bp) and the value of $4.87 \times 10^{9}$ is derived using the following formula: Avogadro's constant $\left(6.02 \times 10^{23}\right)$ / PCR product size $(187 \mathrm{bp}$ for $\mathrm{hB} 2 \mathrm{M}) \times$ molecular weight of ds DNA $(660 \mathrm{Da} / \mathrm{bp})$. 
The PCR product was diluted into six serial dilutions to prepare a stock solution of each of hMito and hB2M PCR products.

\section{Real-Time Quantitative PCR}

Using QuantiTect SYBR Green PCR Kit (Qiagen) the template DNA was prepared, and the dilution standards were used for qPCR to determine the amount of mtDNA and nuclear DNA in the sample. The threshold values were plotted against concentration (Supplementary Figure 1).

\section{Detection of DNA Fragmentation}

Apoptotic DNA fragmentation was analyzed using agarose gel electrophoresis and DNA laddering assay to exclude samples with a high apoptotic rate. Apoptotic cells are characterized by chromatin cleavage and nucleosomesized (200-bp) and multiples thereof (400, 800, etc.) DNA fragments formation.

\section{Statistical Analyses}

The sample size was estimated by MedCalc Statistical Software version 12.4 (MedCalc Software bvba, Ostend, Belgium; 2013). All data were revised for completeness and accuracy, and pre-coded data were entered on the computer using the Statistical Package of Social Science software program, version 22 (SPSS) to be statistically analyzed. Data were summarized using (Mean and SEM for quantitative variables, Number, and percent for qualitative variable), comparison between quantitative variables was done using independent $t$-test for normally distributed quantitative variables and nonparametric Mann-Whitney tests for non-normally distributed quantitative variables, and Receiver Operator Characteristic curve (ROC) was used to find out the best cut off and validity of certain variable. $P$-value of less than or equal to 0.05 is considered significant.

\section{Bioinformatics Study}

Bioinformatics study of microarray data set available on the public database Gene Expression Omnibus (GEO) repository (accession number: GSE5850) was used to investigate the activity of mitochondrial genes in PCOS oocytes compared to control. ${ }^{26}$

\section{Results}

\section{Baseline Characteristics}

The age (mean \pm SEM) of the control group was 26.52 \pm 0.35 years, while in PCOS patients it was $26.97 \pm 0.22$ years. The BMI (mean \pm SEM) of the control group was $23.45 \pm 0.29 \mathrm{~kg} / \mathrm{m}^{2}$ while in PCOS patients it was 23.16 $\pm 0.16 \mathrm{~kg} / \mathrm{m}^{2}$. There was no significant difference between the groups regarding the distribution of age and BMI. The hormonal profile of control and PCOS women was measured and is listed in (Table 1).

The number of days of stimulation and induction dose showed no significant difference between control and PCOS groups. Additionally, the number of mature, fertilized, and good embryos for transfer showed no significant difference between control and PCOS groups. However, the number of retrieved oocytes was significantly higher in PCOS compared to the control group (Table 2).

\section{mtDNA/gDNA Ratio and Clinical Pregnancy Rate}

The clinical pregnancy rate was assessed 4 weeks after ET by detection of intrauterine gestational sac using transvaginal ultrasound (Figure 1).

The control group showed non-statistically significant higher pregnancy rates than the PCOS, frozen ET, and fresh ET with mean $\pm \operatorname{SEM}(0.55 \pm 0.09,0.44 \pm 0.05,0.48$ \pm 0.07 , and $0.40 \pm 0.07$, respectively). Also, the frozen ET group showed non-statistically significant higher pregnancy rates than the fresh embryo group $(\mathrm{P}=0.43)$.

Table I Comparison Between Control and PCOS Women

\begin{tabular}{|l|l|l|l|}
\hline Mean \pm SEM & Control $(\mathbf{N}=33)$ & PCOS Women $(\mathbf{N}=100)$ & P-value \\
\hline LH $(\mathrm{mlU} / \mathrm{mL})$ & $4.2 \pm 0.17$ & $7.3 \pm 0.12$ & $<0.000 I^{* * *}$ \\
FSH $(\mathrm{mlU} / \mathrm{mL})$ & $7.5 \pm 0.19$ & $5.6 \pm 0.09$ & $<0.000 I^{* * *}$ \\
LH/FSH ratio & $0.57 \pm 0.03$ & $1.4 \pm 0.03$ & $<0.000 I^{* * *}$ \\
Estradiol $(\mathrm{pg} / \mathrm{mL})$ & $49.8 \pm 1.06$ & $61.7 \pm 0.78$ & $<0.000 I^{* * *}$ \\
\hline
\end{tabular}

Note: ***P<0.00I. 
Table 2 Characteristic Data of Ovarian Stimulation Protocol and Ovarian Response to Stimulation

\begin{tabular}{|c|c|c|c|c|}
\hline \multirow[t]{2}{*}{ Mean \pm SEM } & \multirow[t]{2}{*}{ Control $(\mathrm{N}=33)$} & \multicolumn{2}{|c|}{ PCOS Women $(\mathrm{N}=100)$} & \multirow[t]{2}{*}{$\mathbf{P}$ value } \\
\hline & & $\begin{array}{l}\text { Fresh ET } \\
(\mathrm{N}=50)\end{array}$ & Frozen ET $(\mathrm{N}=50)$ & \\
\hline \multirow[t]{2}{*}{ Number of days of stimulation } & \multirow[t]{2}{*}{$13.12 \pm 0.19$} & \multicolumn{2}{|l|}{$13.45 \pm 0.12$} & 0.15 \\
\hline & & $13.2 \pm 0.16$ & $13.7 \pm 0.16$ & $>0.05^{*}$ \\
\hline \multirow[t]{2}{*}{ Total dose of induction (IU/I) } & \multirow[t]{2}{*}{$1872.7 \pm 24.17$} & \multicolumn{2}{|l|}{$1930.7 \pm 31.08$} & 0.14 \\
\hline & & $1842.9 \pm 52.7$ & $2018.5 \pm 28.5$ & $>0.05^{*}$ \\
\hline \multirow[t]{2}{*}{ Number of oocytes retrieved } & \multirow[t]{2}{*}{$14.18 \pm 0.24$} & \multicolumn{2}{|l|}{$17.4 \pm 0.3$} & $>0.0001 * * *$ \\
\hline & & $17.46 \pm 0.44$ & $17.4 \pm 0.42$ & 0.92 \\
\hline \multirow[t]{2}{*}{ Number of mature oocytes } & \multirow[t]{2}{*}{$10.24 \pm 0.28$} & \multicolumn{2}{|l|}{$10.57 \pm 0.19$} & 0.36 \\
\hline & & $10.58 \pm 0.27$ & $10.56 \pm 0.26$ & 0.96 \\
\hline \multirow[t]{2}{*}{ Number of fertilized oocytes } & \multirow[t]{2}{*}{$8.94 \pm 0.26$} & \multicolumn{2}{|l|}{$9.3 \pm 0.18$} & 0.31 \\
\hline & & $9.3 \pm 0.23$ & $9.3 \pm 0.29$ & 1.0 \\
\hline \multirow[t]{2}{*}{ Number of good embryos for transfer } & \multirow[t]{2}{*}{$6.4 \pm 0.22$} & \multicolumn{2}{|l|}{$6.4 \pm 0.17$} & 0.99 \\
\hline & & $6.26 \pm 0.21$ & $6.58 \pm 0.26$ & 0.37 \\
\hline
\end{tabular}

Notes: $* \mathrm{P} \leq 0.05$ and $* * * \mathrm{P}<0.001$

mtDNA/gDNA ratio is statistically compared in clinical pregnancy positive and clinical pregnancy negative group as presented in (Figure 2).

Measurement of mtDNA/gDNA ratio in all subjects showed that the clinical pregnancy positive group is significantly higher than the clinical pregnancy negative group (276.3 \pm 20.42 versus $74.39 \pm 12.96$, respectively, ${ }^{* * *} \mathrm{P}<0.0001$ ), and is significantly higher in the control group $(447.3 \pm 22.99$ versus $275.8 \pm 25.22$, respectively, $* * * \mathrm{P}<0.001$ ). It is also significantly higher in the frozen ET group (95.95 \pm 9.94 versus 27.09 \pm 3.98 , respectively, $\left.{ }^{* * *} \mathrm{P}<0.0001\right)$. This ratio at clinically pregnant patients of the fresh ET group is significantly higher than clinically pregnant negative group $(340.78 \pm 18.28$ versus $33.49 \pm 3.6$, respectively, ${ }^{* * *} \mathrm{P}<0.0001$ ).

ROC curve is assisted to evaluate the predictive value of the mtDNA/gDNA ratio in embryo culture media for clinical pregnancy rate in the control group and PCOS group (Figure 3).

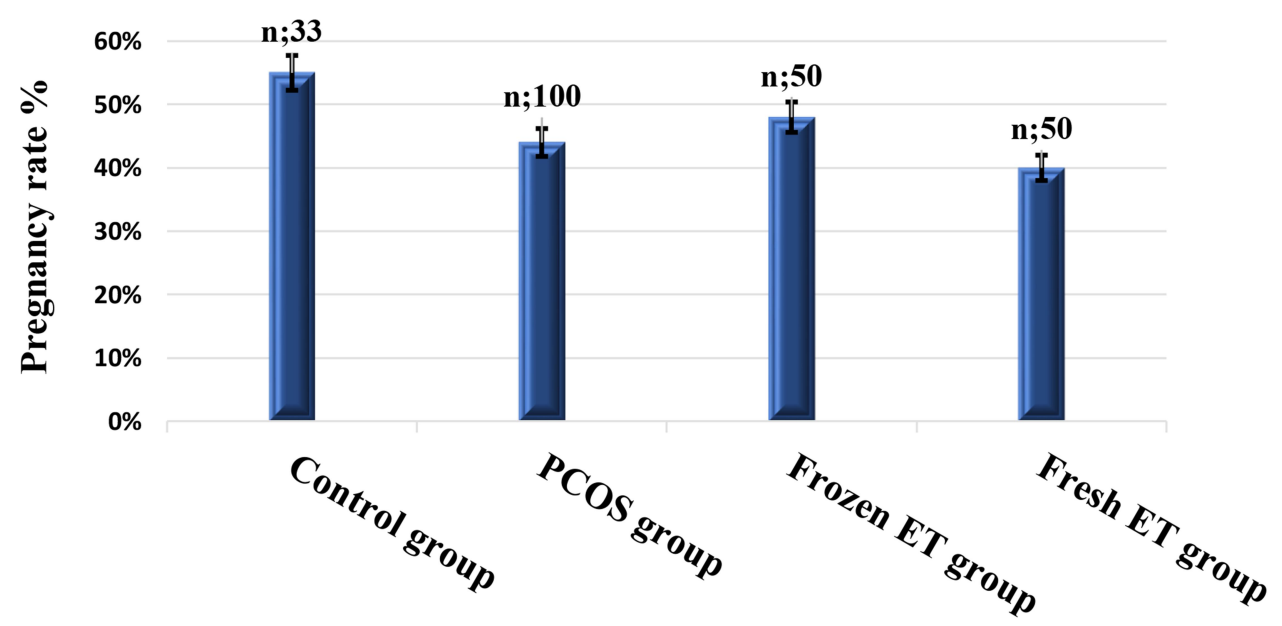

Figure I Bar graphs comparing clinical pregnancy rate in the studied groups. (Mann-Whitney U-test for unpaired non-parametric data). 


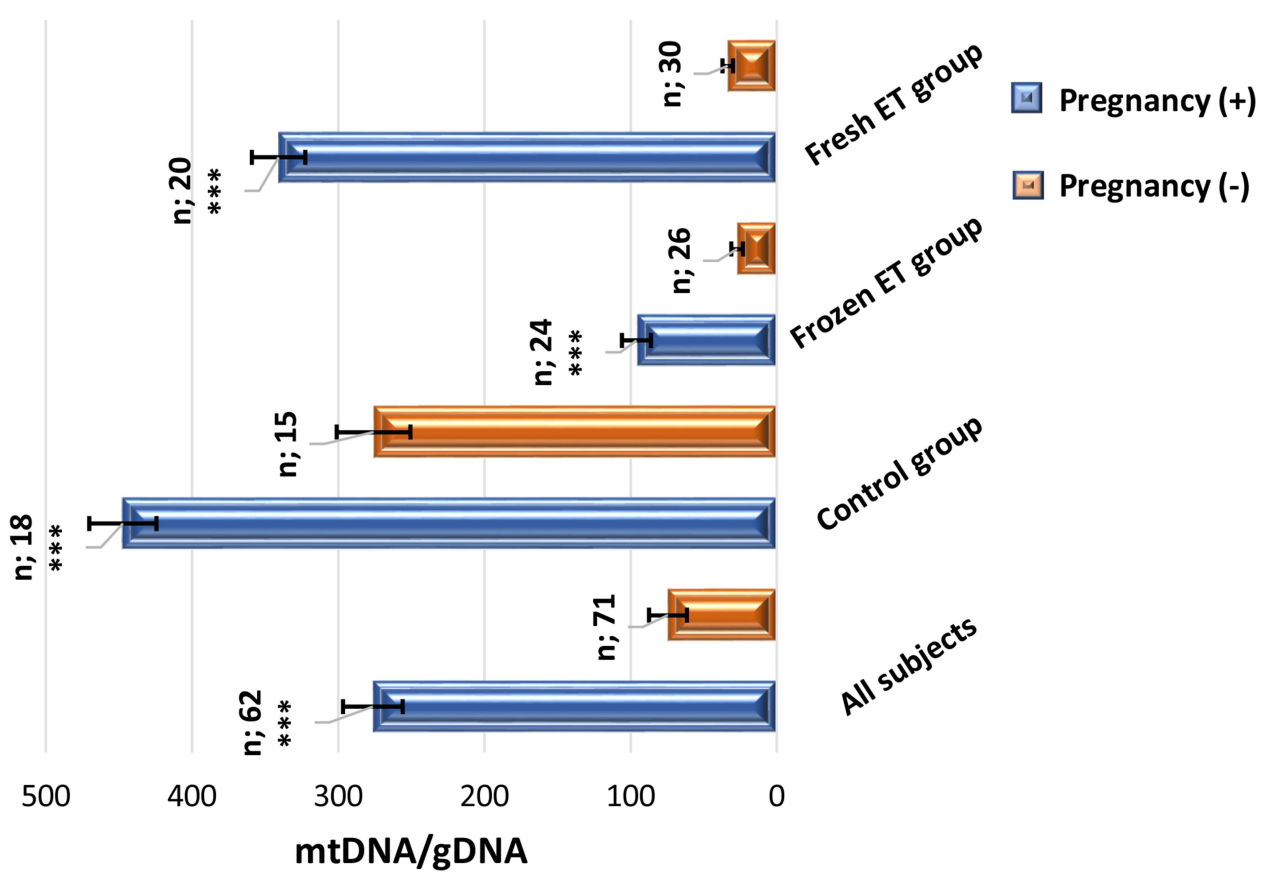

Figure 2 Bar graphs comparing mtDNA/gDNA ratio of embryo culture media regarding clinical pregnancy rate in different groups. (Mann-Whitney U-test for unpaired nonparametric data). ${ }^{*} * *<<0.001$.

\section{Fresh versus Frozen ET Groups}

mtDNA/gDNA ratio (Figure 4) and severe OHSS rate (Figure 5) were compared between the studied groups. mtDNA/gDNA ratio of the control group is significantly higher than the PCOS, frozen, and fresh ET group with mean \pm SEM $(369.34 \pm 22.53,108.3 \pm 12.8260 .14 \pm 7.16$, and $156.41 \pm 22.78$, respectively, $* * * \mathrm{P}<0.0001$ ). Also, it was high, though not statistically significant, in the fresh ET than frozen ET group ( $\mathrm{P}=0.06)$.

Severe OHSS rate is high, though not statistically significant, in the fresh ET group than the frozen ET group $(0.10 \pm 0.04$ versus $0.04 \pm 0.03$, respectively, $\mathrm{P}=0.24)$.

The linear regression showed that $\mathrm{mtDNA} / \mathrm{gDNA}$ ratio in embryo culture media was inversely proportional to basal LH and LH/FSH (Figure 6) of the PCOS group with $\mathrm{r}^{2}(0.5685$ and 0.3166 , respectively, $* * * \mathrm{P}<0.0001)$.

\section{DNA Fragmentation}

No fragmentation was detected in all samples included in the study (showed no ladder pattern of 180-200 bp) thus did not have a high rate of apoptosis.

\section{Bioinformatics Study}

Data set (GSE5850) analysis was performed to get the relation between mitochondrial activity and the successful
IVF. Differentially expressed genes were detected having corrected $P$-value $\leq 0.05$ (Bonferroni correction). Per our analysis, the expression of mitochondrial genes in six normal and six PCOS oocytes found eight genes that were significantly dysregulated that involved in the mtDNA transcription and translation, oxidative phosphorylation activity, and ATP generation in PCOS oocytes. These results explain the low embryo quality and pregnancy rate in the PCOS group than control (Table 3).

\section{Discussion}

In routine IVF practices, embryo selection is mainly based on morphological criteria examined by light microscopy. ${ }^{27}$ However, the morphologically good embryos do not indicate good functional status as $30-50 \%$ of these embryos might be genetically abnormal. ${ }^{28}$ Preimplantation genetic assessment requires embryo cell biopsy which is a highly invasive method and requires highly skilled personnel. ${ }^{29}$ Recent studies reported that embryo releases its genetic material in culture media so genetic analysis of this media can be used as a non-invasive assessment of embryo quality. ${ }^{21}$ The fertilization ability of an oocyte and quality of the resulting embryo is dependent on its ATP content. ${ }^{30}$

In the present study, the release of both mitochondrial and genomic DNA has been demonstrated in the culture media of embryos. It was observed that mtDNA/gDNA 

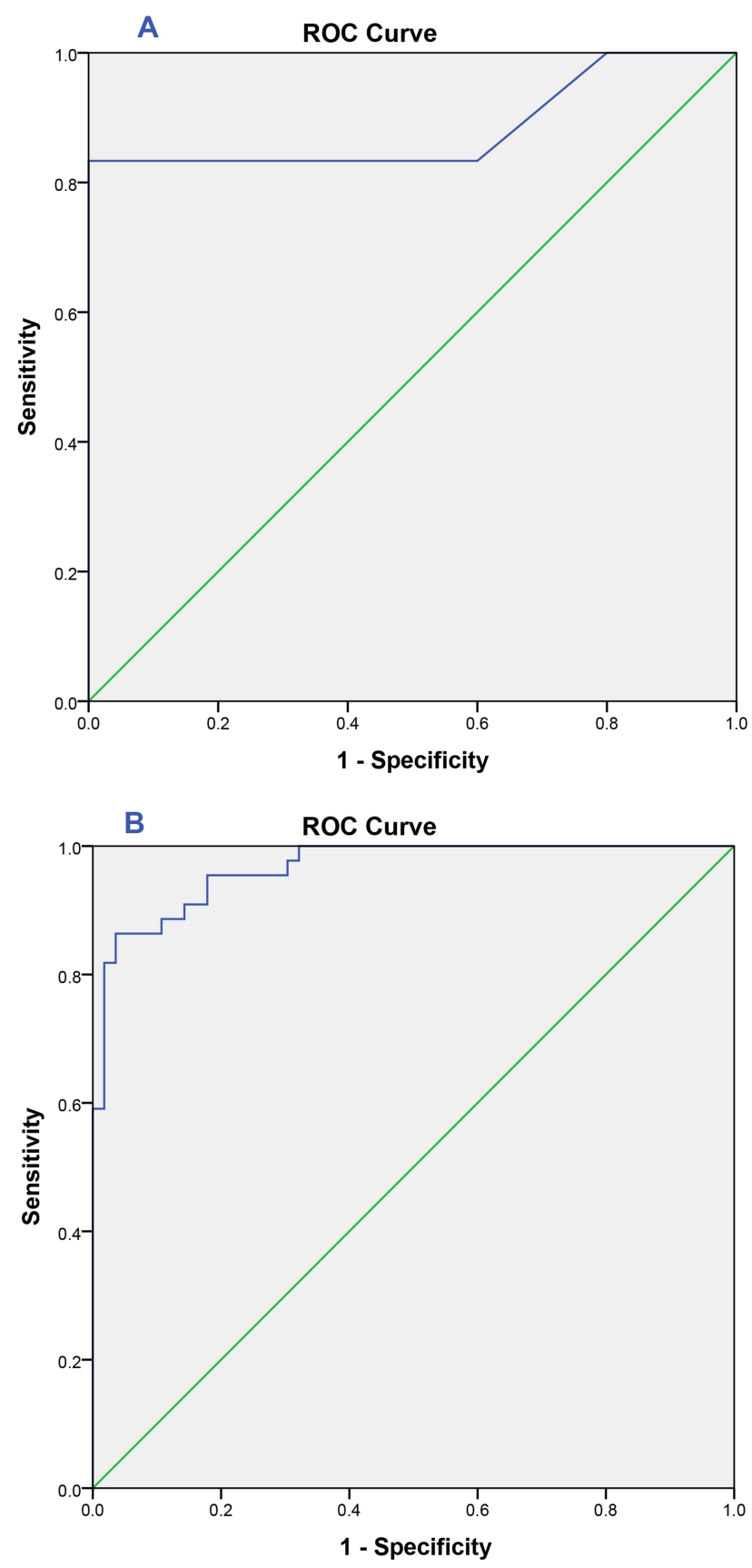

Figure 3 ROC curve of mtDNA/gDNA ratio: (A) Healthy control group undergoing IVF: area under the curve $(A \cup C)=0.89$ [0.76-I.0], cutoff value $=386.29$ with sensitivity $=83 \%$ and specificity $=100 \%$. (B) PCOS group undergoing IVF: area under the curve $(A \cup C)=0.97$ [0.94-0.99], cutoff value $=68.59$ with sensitivity $=86 \%$ and specificity $=96 \%$.

ratio reflects embryo quality and correlates with pregnancy rate, as this ratio was significantly higher in the pregnant group in comparison to the non-pregnant group (276.3 \pm 20.42 versus $74.39 \pm 12.96$, respectively, $* * * \mathrm{P}<0.0001$ ) (Figure 2).

Our observations go hand in hand with the literature review, which reported that an embryo is permeable to
DNA as it is surrounded by a highly porous membrane (zona pellucida) that allows the migration of dsDNA particles. ${ }^{31}$ Previous studies found that only $37 \%$ of analyzed embryo culture media samples contain gDNA while almost all of them had mtDNA in a higher concentration due to its cytoplasmic localization, which indicates that the presence of DNA in the media does not always represent apoptosis or necrosis. "mtDNA bottleneck theory" stated that the total amount of maternally inherited mtDNA in the oocytes split between blastomeres during each division so that high mtDNA oocyte content is associated with better embryo development and implantation. ${ }^{33}$ It is believed that mtDNA content in oocytes increases during oogenesis and rapidly decreases in embryo cell development, without any mtDNA replication. ${ }^{34}$ It was also reported that euploid embryos with low viability and implantation potential contain a high amount of mtDNA. ${ }^{35}$ Therefore, embryos with low quality are known to have a high cytoplasmic content of mtDNA, increased gDNA degradation over time, and they release a small amount of mtDNA in the embryo culture media and keep most of the oocyte-derived mtDNA to support embryo development. So, embryo culture media mtDNA/gDNA ratio can reflect the overall embryonic mtDNA content and subsequently embryo quality. ${ }^{36}$

PCOS is an endocrine-metabolic disorder associated with an imbalance between extra- and intra-ovarian factors that negatively affect oocyte maturation and embryonic development. ${ }^{4}$ Hypersecretion of LH during the follicular phase may inhibit FSH function resulting in premature granulosa cell lutelization and premature oocyte maturation by inhibition of oocyte maturation inhibitors that impair embryo quality. ${ }^{37}$

Our results also revealed that mtDNA/gDNA ratio was significantly lower in PCOS when compared to the control group in embryo culture media $(* * * \mathrm{P}<0.0001)$ (Figure 4$)$. This observation may reflect lower embryo quality in PCOS patients and lower chances of achieving pregnancy. Therefore, extensive research should be directed toward improving the pregnancy rates in PCOS by evaluating the different strategies as the freeze-all policy which was targeted in our study.

Reduced mtDNA/gDNA ratio may reflect poor mitochondrial antioxidant activity that may lead to cell apoptosis. Embryos that successfully develop into blastocysts exhibited a significantly higher mtDNA/gDNA ratio in the culture medium compared with those that arrest. ${ }^{21}$ 


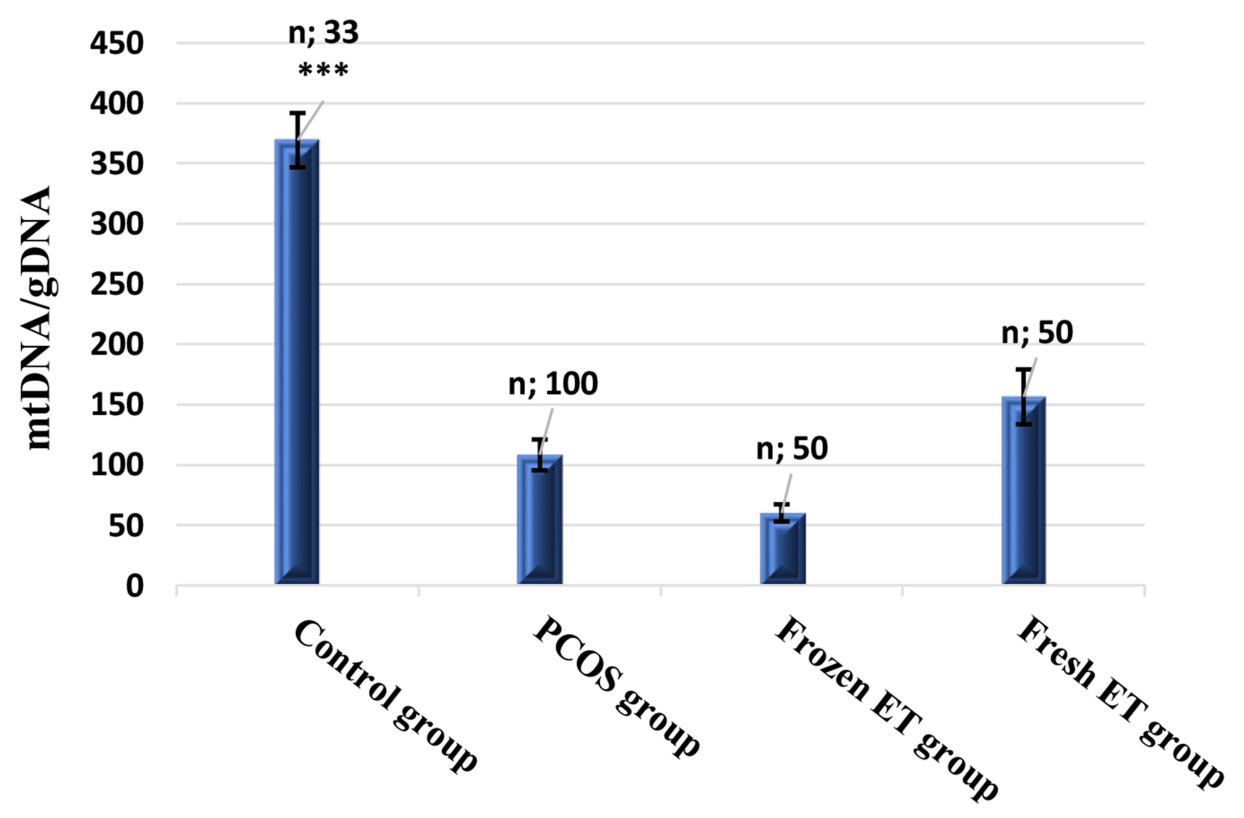

Figure 4 Bar graphs comparing mtDNA/gDNA ratio of embryo culture media in different studied groups. (Mann-Whitney U-test for unpaired non-parametric data). $* * * \mathrm{P}<0.001$.

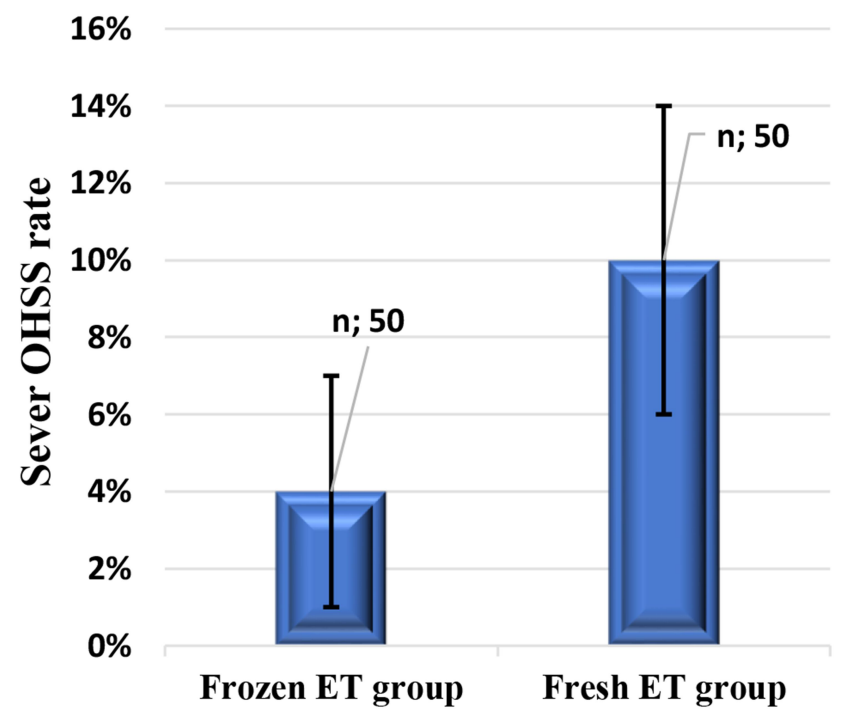

Figure 5 Bar graphs comparing severe OHSS rate in fresh and frozen ET groups.

The data set (GSE5850) analysis showed changes in the expression of mitochondrial genes involved in the mtDNA transcription and translation, oxidative phosphorylation activity, and ATP generation in PCOS oocytes. In our analysis, RHEB gene was significantly upregulated while ATP5B, NADK2, NRF1, TFAM, POLRM, TFB2M, and MTRF1 genes were significantly downregulated (Table 3).

TFAM is thought to enhance transcription by interaction with POLMT and NRF-1, NRF-2. Low levels of mitochondrial TFAM are associated with mtDNA depletion in mice during embryonic stages. ${ }^{38}$

Mitochondrial ATP synthase synthesizes ATP from ADP in the mitochondrial matrix using the energy provided by the proton electrochemical gradient. $^{39}$ Dysregulation in the expression of genes encoding ATP synthase subunits affects the amount of ATP production which is essential for embryo viability.

Interactions between PGC- $1 \alpha$ and NRF1 increase the transcription of nuclear genome-encoded genes for electron transport chain proteins. ${ }^{40}$ PGC-1 $\alpha / \mathrm{NRF} 1$ also increases the transcription of TFAM and the promotion of transcription of the mitochondrial genome-encoded genes. ${ }^{41}$ In PCOS oocytes, downregulation of the NRF1 gene decreases the nuclear expression of mtDNA transcription factors includes TFAM, TFB2M, and POLRMT that are found to be downregulated in PCOS oocytes, which subsequently decrease the production of respiratory subunits encoded by mtDNA.

RHEB is a mitochondrial autophagic receptor that contributes to the mitochondrial autophagy. Thus, Dysregulation of this gene affects RHEB -dependent mitophagy that maintains optimal mitchondrial energy production which is essential for embryo viability. ${ }^{42}$

Our study shows that pregnancy rate in the frozen ET group was not significantly higher than fresh ET. 

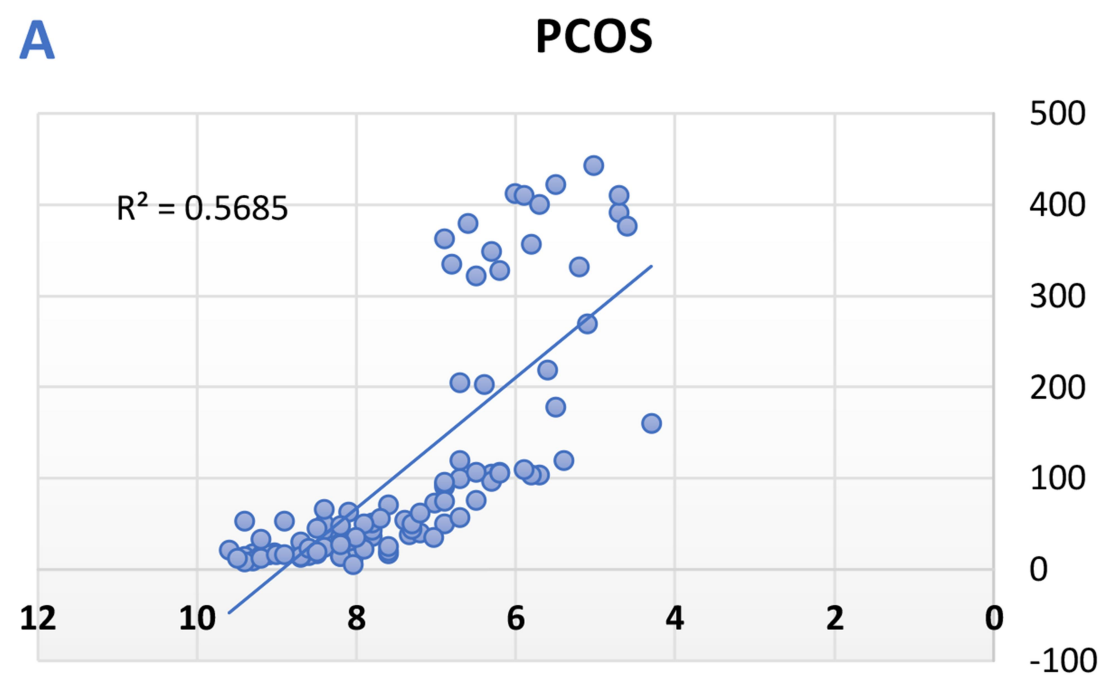

\section{LH}

B

\section{PCOS}

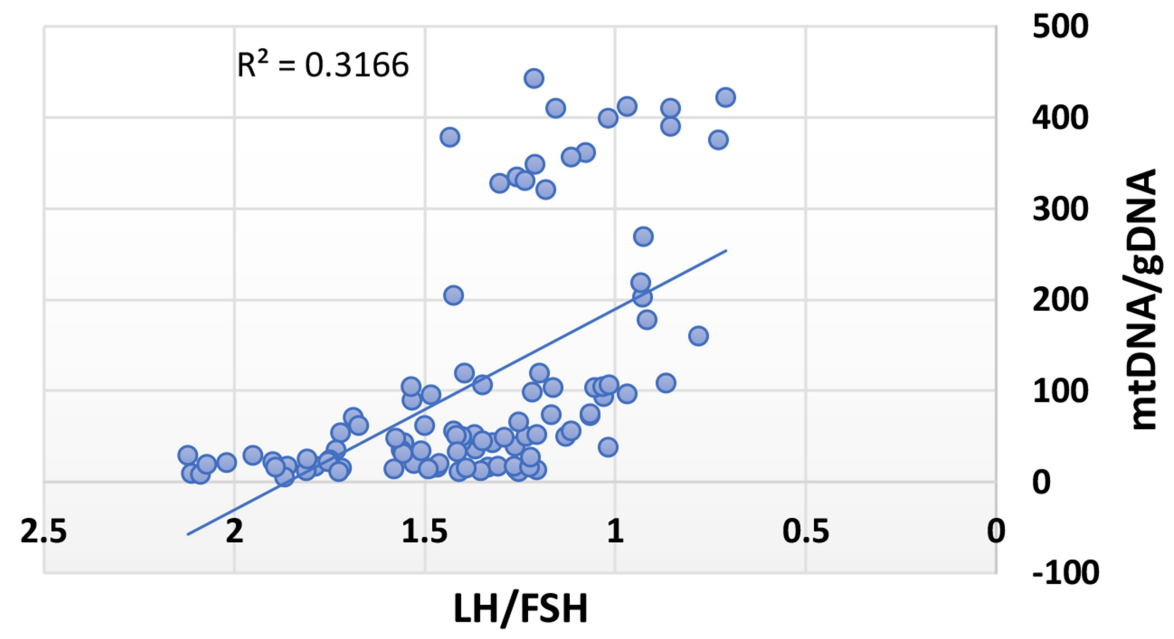

Figure 6 The linear regression of mtDNA/gDNA ratio and (A) basal LH level of the PCOS group, (B) basal LH/FSH of the PCOS group.

Moreover, the rate of OHSS cases associated with fresh ET was higher than frozen ET candidates, yet this difference was not statistically significant.

Indeed, in vitro fertilization success is dependent on several factors such as the number of embryos transferred, the quality of each embryo transferred, the integrity of the endometrium, and endometrial receptivity. ${ }^{43}$ Controlled ovarian stimulation has been found to create a negative effect on endometrium receptivity rather than on oocyte quality. ${ }^{44}$ This effect is due to the presence of elevation in serum progesterone and estrogen levels during the follicular phase. ${ }^{45}$ Estrogen elevation is combined with LH secretion that stimulates progesterone production and inhibits ovulation. Additionally, elevated estrogen upregulates progesterone receptors in the endometrium compared to the natural cycle that increases the maturation of the endometrium. ${ }^{46}$ Embryo cryopreservation has become a common clinical IVF practice. It helps in decreasing the number of embryos transferred and the risks related to OHSS. ${ }^{47}$ Roque and collaborators reported that the frozen ET group showed significantly higher IVF outcomes compared to the fresh ET group. ${ }^{7}$ The ongoing pregnancy rate in the frozen ET strategy was higher but not significant in comparison to fresh ET protocol. ${ }^{9}$

On the other hand, the freeze-thaw process lowers the embryonic viability of animal embryos (Bos indicus) as it 
Table 3 Significantly Dysregulated Mitochondrial Genes with the Observed Fold Change with q-value $\leq 0.05$ in PCOS Oocytes Compared to Control (GSE5850)

\begin{tabular}{|l|l|l|l|}
\hline Gene Symbol & Gene Names & Fold Change & q-value \\
\hline ATP5B & ATP synthase, H+ transporting, mitochondrial FI complex, beta subunit. & $-I .887072$ & $0.05087 I^{*}$ \\
NADK2 & NAD kinase 2, mitochondrial & $-6.61603 I$ & $0.008472^{* *}$ \\
RHEB & Ras homolog enriched in brain & 1.78032 & $0.027776^{*}$ \\
NRFI & Nuclear respiratory factor I & -2.716919 & $0.03619^{*}$ \\
TFAM & Transcription factor A, mitochondrial & -1.275446 & $0.0467238^{*}$ \\
POLRMT & RNA polymerase mitochondrial & -1.790267 & $0.042075^{*}$ \\
TFB2M & Transcription factor B2, mitochondrial & -2.230954 & $0.034842^{*}$ \\
MTRFI & Mitochondrial translational release factor I & -2.00303 & $0.040936 *$ \\
\hline
\end{tabular}

Notes: *q-value $\leq 0.05$ and $* *$-value $\leq 0.01$.

increases levels of ROS and cell apoptosis. ${ }^{48}$ A study reported that in frozen semen, the concentration of free radicals increases. ${ }^{49}$ The freezing process causes the hypothermia-hypoxia phenomenon, which is characterized by the reduction of ATP levels and is associated with the decoupling of the internal mitochondrial membrane. This causes an increase in oxygen levels and subsequent elevation of superoxide free radical level, which promote the formation of hydrogen peroxide and hydroxide free radical. $^{50}$

To summarize, the mtDNA/gDNA ratio in embryo culture media of PCOS women undergoing IVF is significantly higher with pregnancy. Thus, it presents a possible predictive marker for non-invasive evaluation of the quality of morphologically good embryos and pregnancy rate (sensitivity $86 \%$ and specificity $96 \%$ ) (Figure 3). Furthermore, in fresh embryo culture media, mtDNA/gDNA ratio is higher, though not statistically significant, in comparison with the frozen embryo. This is probably because of the freezing and thawing that causes an increase in oxidative stress in the media which may affect the embryo viability.

\section{Conclusion}

Our data show that mtDNA/gDNA ratio in embryo culture media can be used as a useful marker of good embryo quality and subsequently higher pregnancy rate.

\section{Abbreviations}

ET, embryos transfer; PCOS, polycystic ovary syndrome; OHSS, ovarian hyperstimulation syndrome; ICSI, intracytoplasmic sperm injection; r-FSH, recombinant FSH; PCR, polymerase chain reaction; SPSS, Statistical Package of Social Science; ROC, receiver operator characteristic curve; GEO, Gene Expression Omnibus; AUC, area under the curve; ROS, reactive oxygen species; ARE, antioxidant response element; ND1, NADH: ubiquinone oxidoreductase subunit 1; CO I, cytochrome c oxidase subunit 1.

\section{Data Sharing Statement}

The authors are not intending to share individual deidentified participant data.

\section{Acknowledgments}

We acknowledge the effort of all workers in the IVF unit at Ganna IVF center, Dr. Reham Hassan Mekky, Pharmacognosy Department, Egyptian Russian University, for her help in graph drawing and editing, and to Mohamed Badawy, Neurobiology and behavior department, Stony Brook University for his help in proofreading, and editing.

\section{Funding}

This research received no external funding.

\section{Disclosure}

The author reports no conflict of interest in this work.

\section{References}

1. Legro RS. Evaluation and treatment of polycystic ovary syndrome. Endotext. 2017.

2. Angin P, Yoldemir T, Atasayan K. Quality of life among infertile PCOS patients. Arch Gynecol Obstet. 2019;300(2):461-467. doi:10.1007/s00404-019-05202-z

3. Wong KM, van Wely M, Mol F, Repping S, Mastenbroek S. Fresh versus frozen embryo transfers in assisted reproduction. Cochrane Database Syst Rev. 2017;(3). doi:10.1002/14651858.CD011184.pub2

4. Qiao J, Feng HL. Extra-and intra-ovarian factors in polycystic ovary syndrome: impact on oocyte maturation and embryo developmental competence. Hum Reprod Update. 2010;17(1):17-33.

5. Fatemi HM, Garcia-Velasco J. Avoiding ovarian hyperstimulation syndrome with the use of gonadotropin-releasing hormone agonist trigger. Fertil Steril. 2015;103(4):870-873. doi:10.1016/j. fertnstert.2015.02.004 
6. Huang Y, Wang E-Y, Du Q-Y, et al. Progesterone elevation on the day of human chorionic gonadotropin administration adversely affects the outcome of IVF with transferred embryos at different developmental stages. Reprod Biol Endocrinol. 2015;13(1):82. doi:10.1186/s12958015-0075-3

7. Roque M, Lattes K, Serra S, et al. Fresh embryo transfer versus frozen embryo transfer in in vitro fertilization cycles: a systematic review and meta-analysis. Fertil Steril. 2013;99(1):156-162. doi:10.1016/j.fertnstert.2012.09.003

8. Wong KM, Mastenbroek S, Repping S. Cryopreservation of human embryos and its contribution to in vitro fertilization success rates. Fertil Steril. 2014;102(1):19-26. doi:10.1016/j.fertnstert.2014.05.027

9. Chen Z-J, Shi Y, Sun Y, et al. Fresh versus frozen embryos for infertility in the polycystic ovary syndrome. $N$ Engl $\mathrm{J} \mathrm{Med}$. 2016;375(6):523-533. doi:10.1056/NEJMoa1513873

10. Coates A, Kung A, Mounts E, et al. Optimal euploid embryo transfer strategy, fresh versus frozen, after preimplantation genetic screening with next generation sequencing: a randomized controlled trial. Fertil Steril. 2017;107(3):723-730. e723. doi:10.1016/j.fertnste rt.2016.12.022

11. Stormlund S, Schmidt L, Bogstad J, et al. Patients' attitudes and preferences towards a freeze-all strategy in ART treatment. Hum Reprod. 2019;34(4):679-688. doi:10.1093/humrep/dez006

12. Zhu Q, Chen Q, Wang L, et al. Live birth rates in the first complete IVF cycle among 20687 women using a freeze-all strategy. Hum Reprod. 2018;33(5):924-929. doi:10.1093/humrep/dey044

13. Teh WT, Polyakov A, Garrett C, Edgar D, Mcbain J, Rogers PAW. Reduced live birth rates in frozen versus fresh single cleavage stage embryo transfer cycles: a cross-sectional study. Int J Reprod Biomed. 2020;18(7):491.

14. Cecchino GN, Garcia-Velasco JA. Mitochondrial DNA copy number as a predictor of embryo viability. Fertil Steril. 2019;111(2):205-211. doi:10.1016/j.fertnstert.2018.11.021

15. Capalbo A, Rienzi L, Cimadomo D, et al. Correlation between standard blastocyst morphology, euploidy and implantation: an observational study in two centers involving 956 screened blastocysts. Hum Reprod. 2014;29(6):1173-1181. doi:10.1093/humrep/deu033

16. Katz-Jaffe MG, McReynolds S. Embryology in the era of proteomics. Fertil Steril. 2013;99(4):1073-1077. doi:10.1016/j.fertnster t.2012.12.038

17. May-Panloup P, Chrétien MF, Savagner F, et al. Increased sperm mitochondrial DNA content in male infertility. Hum Reprod. 2003;18(3):550-556. doi:10.1093/humrep/deg096

18. Anderson S, Bankier AT, Barrell BG, et al. Sequence and organization of the human mitochondrial genome. Nature. 1981;290 (5806):457-465. doi:10.1038/290457a0

19. Kaneda H, Hayashi J, Takahama S, Taya C, Lindahl KF, Yonekawa H. Elimination of paternal mitochondrial DNA in intraspecific crosses during early mouse embryogenesis. Proc Natl Acad Sci. 1995;92(10):4542-4546. doi:10.1073/pnas.92.10.4542

20. Seyedhassani SM, Houshmand M, Kalantar SM, Modabber G, Aflatoonian A. No mitochondrial DNA deletions but more D-loop point mutations in repeated pregnancy loss. J Assist Reprod Genet. 2010;27(11):641-648. doi:10.1007/s10815-010-9435-2

21. Stigliani S, Persico L, Lagazio C, Anserini P, Venturini P, Scaruffi P. Mitochondrial DNA in day 3 embryo culture medium is a novel, non-invasive biomarker of blastocyst potential and implantation outcome. Mol Hum Reprod. 2014;20(12):1238-1246. doi:10.1093/ molehr/gau086

22. ESHRE TR, Group A-SPCW. Revised 2003 consensus on diagnostic criteria and long-term health risks related to polycystic ovary syndrome. Fertil Steril. 2004;81(1):19-25. doi:10.1016/j. fertnstert.2003.10.004

23. Alfaraj S, Alzaher F, Alshwaiaer S, Ahmed A. Pregnancy outcome of day 3 versus day 5 embryo transfer: a retrospective analysis. Asian Pac J Reprod. 2019;2:89-92.
24. $\mathrm{Hu}$ Y, Maxson WS, Hoffman DI, et al. Maximizing pregnancy rates and limiting higher-order multiple conceptions by determining the optimal number of embryos to transfer based on quality. Fertil Steril. 1998;69(4):650-657. doi:10.1016/S0015-0282(98)00024-7

25. Ajaz S, Czajka A, Malik A. Accurate measurement of circulating mitochondrial DNA content from human blood samples using real-time quantitative PCR. Mitochondrial Med. 2015;117-131.

26. Wood JR, Dumesic DA, Abbott DH, Strauss III JF. Molecular abnormalities in oocytes from women with polycystic ovary syndrome revealed by microarray analysis. J Clin Endocrinol Metab. 2007;92(2):705-713. doi:10.1210/jc.2006-2123

27. Gardner DK, Lane M, Stevens J, Schlenker T, Schoolcraft WB. Blastocyst score affects implantation and pregnancy outcome: towards a single blastocyst transfer. Fertil Steril. 2000;73 (6):1155-1158. doi:10.1016/S0015-0282(00)00518-5

28. Fragouli E, Alfarawati S, Spath K, Wells D. Morphological and cytogenetic assessment of cleavage and blastocyst stage embryos. Mol Hum Reprod. 2013;20(2):117-126. doi:10.1093/molehr/gat073

29. Scott RT, Upham KM, Forman EJ, Zhao T, Treff NR. Cleavage-stage biopsy significantly impairs human embryonic implantation potential while blastocyst biopsy does not: a randomized and paired clinical trial. Fertil Steril. 2013;100(3):624-630. doi:10.1016/j. fertnstert.2013.04.039

30. Fragouli E, McCaffrey C, Ravichandran K, et al. Clinical implications of mitochondrial DNA quantification on pregnancy outcomes: a blinded prospective non-selection study. Hum Reprod. 2017;32 (11):2340-2347. doi:10.1093/humrep/dex292

31. Chi H-J, Koo -J-J, Choi S-Y, Jeong H-J, Roh S-I. Fragmentation of embryos is associated with both necrosis and apoptosis. Fertil Steril. 2011;96(1):187-192. doi:10.1016/j.fertnstert.2011.04.020

32. Stigliani S, Anserini P, Venturini P, Scaruffi P. Mitochondrial DNA content in embryo culture medium is significantly associated with human embryo fragmentation. Hum Reprod. 2013;28(10):2652-2660. doi:10.1093/humrep/det314

33. Desquiret-Dumas V, Clément A, Seegers V, et al. The mitochondrial DNA content of cumulus granulosa cells is linked to embryo quality. Hum Reprod. 2017;32(3):607-614. doi:10.1093/humrep/dew341

34. Hashimoto S, Morimoto N, Yamanaka M, et al. Quantitative and qualitative changes of mitochondria in human preimplantation embryos. J Assist Reprod Genet. 2017;34(5):573-580. doi:10.1007/ s10815-017-0886-6

35. Diez-Juan A, Rubio C, Marin C, et al. Mitochondrial DNA content as a viability score in human euploid embryos: less is better. Fertil Steril. 2015;104(3):534-541. e531. doi:10.1016/j.fertnstert.2015.05.022

36. Stigliani S, Orlando G, Massarotti C, et al. Non-invasive mitochondrial DNA quantification on day 3 predicts blastocyst development: a prospective, blinded, multi-centric study. Mol Hum Reprod. 2019;25(9):527-537. doi:10.1093/molehr/gaz032

37. Santos MA, Kuijk EW, Macklon NS. The impact of ovarian stimulation for IVF on the developing embryo. Reproduction. 2010;139 (1):23-34. doi:10.1530/REP-09-0187

38. Larsson N-G, Wang J, Wilhelmsson H, et al. Mitochondrial transcription factor A is necessary for mtDNA maintenance and embryogenesis in mice. Nat Genet. 1998;18(3):231-236. doi:10.1038/ng0398-231

39. Zeviani M, Di Donato S. Mitochondrial disorders. Brain. 2004;127 (10):2153-2172. doi:10.1093/brain/awh259

40. Wu Z, Puigserver P, Andersson U, et al. Mechanisms controlling mitochondrial biogenesis and respiration through the thermogenic coactivator PGC-1. Cell. 1999;98(1):115-124. doi:10.1016/S00928674(00)80611-X

41. Gordon JW, Rungi AA, Inagaki H, Hood DA. Plasticity in skeletal, cardiac, and smooth muscle: selected contribution: effects of contractile activity on mitochondrial transcription factor A expression in skeletal muscle. J Appl Physiol. 2001;90(1):389-396. doi:10.1152/ japp1.2001.90.1.389 
42. Melser S, Chatelain EH, Lavie J, et al. Rheb regulates mitophagy induced by mitochondrial energetic status. Cell Metab. 2013;17 (5):719-730. doi:10.1016/j.cmet.2013.03.014

43. Milachich T, Shterev A. Are there optimal numbers of oocytes, spermatozoa and embryos in assisted reproduction? JBRA Assist Reprod. 2016;20(3):142. doi:10.5935/1518-0557.20160032

44. Lu X, Chen Q, Fu Y, Ai A, Lyu Q, Kuang YP. Elevated progesterone on the trigger day does not impair the outcome of human menotrophins gonadotrophin and medroxyprogesterone acetate treatment cycles. Sci Rep. 2016;6:31112. doi:10.1038/srep31112

45. Venetis C, Kolibianakis E, Bosdou J, Tarlatzis B. Progesterone elevation and probability of pregnancy after IVF: a systematic review and meta-analysis of over 60000 cycles. Hum Reprod Update. 2013;19 (5):433-457. doi:10.1093/humupd/dmt014

46. Koo HS, Cha SH, Kim HO, et al. A high response to controlled ovarian stimulation induces premature luteinization with a negative impact on pregnancy outcomes in a gonadotropin-releasing hormone antagonist cycle. Clin Exp Reprod Med. 2015;42(4):149-155. doi:10.5653/cerm.2015.42.4.149
47. Shapiro BS, Daneshmand ST, Garner FC, Aguirre M, Hudson C, Thomas S. Evidence of impaired endometrial receptivity after ovarian stimulation for in vitro fertilization: a prospective randomized trial comparing fresh and frozen-thawed embryo transfer in normal responders. Fertil Steril. 2011;96(2):344-348. doi:10.1016/j. fertnstert.2011.05.050

48. López-Damián EP, Jiménez-Medina JA, Alarcón MA, et al. Cryopreservation induces higher oxidative stress levels in Bos indicus embryos compared with Bos taurus. Theriogenology. 2019;143:74-81. doi:10.1016/j.theriogenology.2019.12.001

49. Tatone C, Di Emidio G, Vento M, Ciriminna R, Artini PG. Cryopreservation and oxidative stress in reproductive cells. Gynecol Endocrinol. 2010;26(8):563-567. doi:10.3109/09513591003686395

50. Chatterjee S, Gagnon C. Evidence for the production of oxygen free radicals during freezing/thawing of bull spermatozoa. Mol Reprod Dev. 2001;59(4):451-458. doi:10.1002/mrd.1052

\section{Publish your work in this journal}

Pharmacogenomics and Personalized Medicine is an international, peer-reviewed, open access journal characterizing the influence of genotype on pharmacology leading to the development of personalized treatment programs and individualized drug selection for improved safety, efficacy and sustainability. This journal is indexed on the American Chemical Society's Chemical Abstracts Service (CAS). The manuscript management system is completely online and includes a very quick and fair peer-review system, which is all easy to use. Visit http://www.dovepress.com/testimonials.php to read real quotes from published authors. 